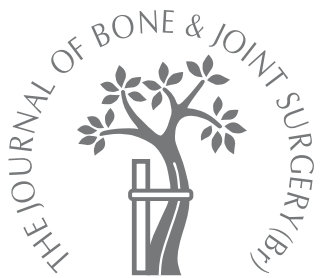

C. W. McBryde,

E. Shears,

J. N. O'Hara,

P. B. Pynsent

From The Royal

Orthopaedic

Hospital,

Birmingham,

England

\title{
Metal-on-metal hip resurfacing in developmental dysplasia
}

\author{
A CASE-CONTROL STUDY
}

Metal-on-metal hip resurfacing was performed for developmental dysplasia in 96 hips in 85 patients, 78 in women and 18 in men, with a mean age at the time of surgery of 43 years (14 to 65). These cases were matched for age, gender, operating surgeon and date of operation with a group of patients with primary osteoarthritis who had been treated by resurfacing, to provide a control group of 96 hips (93 patients). A clinical and radiological follow-up study was performed. The dysplasia group were followed for a mean of 4.4 years (2.0 to 8.5) and the osteoarthritis group for a mean of 4.5 years (2.2 to 9.4). Of the dysplasia cases, 17 (18\%) were classified as Crowe grade III or IV.

There were five (5.2\%) revisions in the dysplasia group and none in the osteoarthritic patients. Four of the failures were due to acetabular loosening and the other sustained a fracture of the neck of femur. There was a significant difference in survival between the two groups ( $p=0.02)$. The five-year survival was $96.7 \%(95 \%$ confidence interval 90.0 to 100$)$ for the dysplasia group and $100 \%$ (95\% confidence interval 100 to 100$)$ for the osteoarthritic group. There was no significant difference in the median Oxford hip score between the two groups at any time during the study.

The medium-term results of metal-on-metal hip resurfacing in all grades of developmental dysplasia are encouraging, although they are significantly worse than in a group of matched patients with osteoarthritis treated in the same manner.

Total hip replacement (THR) in patients with developmental dysplasia presents a challenge to the orthopaedic surgeon. There is considerable variation of both the femoral and acetabular bony anatomy with associated soft-tissue contractures and muscle weakness. ${ }^{1-5}$ The mean age of these patients requiring a THR is relatively low, and so the demands placed on any subsequent arthroplasty can be high. ${ }^{6}$ Resurfacing arthroplasty is an attractive option for younger patients as it preserves the femoral bone stock and provides good stability and a low risk of dislocation owing to the large diameter of the articulation. Historically, hip resurfacing using metal-onpolyethylene had unacceptably high rates of failure when used in developmental dysplasia. ${ }^{7}$ Contemporary resurfacing designs have a metal-on-metal bearing which has a very low wear rate, and good medium-term results have been published in young and active patients treated for osteoarthritis (OA). ${ }^{8-11}$ The use of this type of resurfacing in the treatment of developmental dysplasia is increasing in popularity. ${ }^{12}$ A $96 \%$ survival rate at five years has been reported in a series of patients of whom $70 \%$ had developmental dysplasia of the hip. ${ }^{12}$ In contrast, a separate study has reported disappointing medium-term results, with five femoral failures in 59 hips at a mean of six years. ${ }^{3}$ The aim of our study was to compare the medium-term results of the Birmingham Hip Resurfacing (Smith \& Nephew PLC, London, United Kingdom) in a group of patients with developmental dysplasia matched with a group with OA treated in the same manner.

\section{Patients and Methods}

A consecutive series of patients was studied between July 1997 and July 2004. A computer database is kept at our institution for all resurfacing procedures, containing demographic, diagnostic, surgical, follow-up and radiological details. Analysis of the database identified 96 hips (85 patients) with a diagnosis of OA secondary to developmental dysplasia which were treated during this period with a Birmingham Hip Resurfacing. Over the same period of time, 1099 consecutive Birmingham Hip Resurfacings were also performed where the diagnosis was 'primary' OA. This was the 


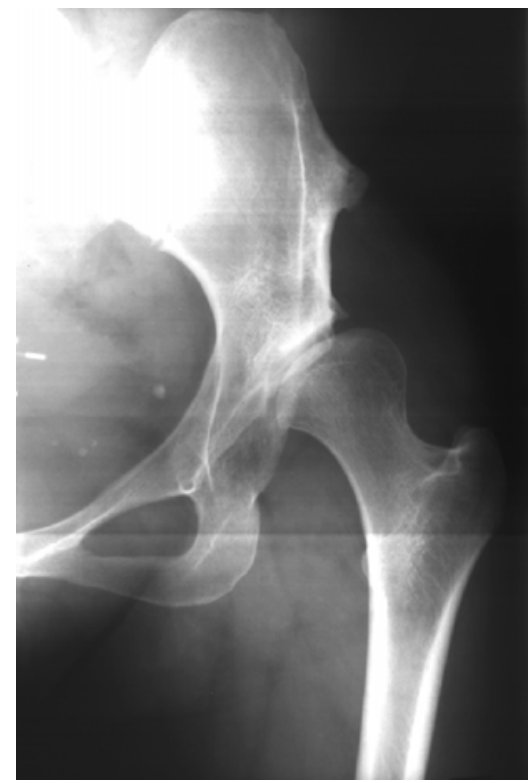

Fig. 1a

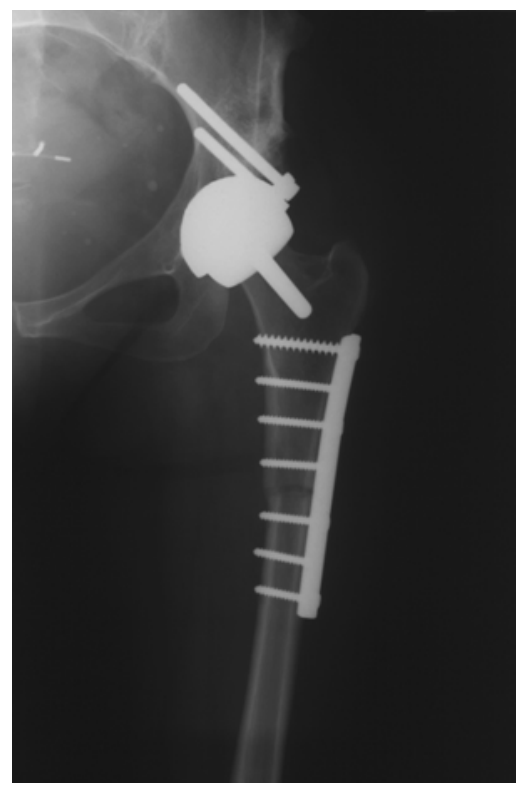

Fig. 1b

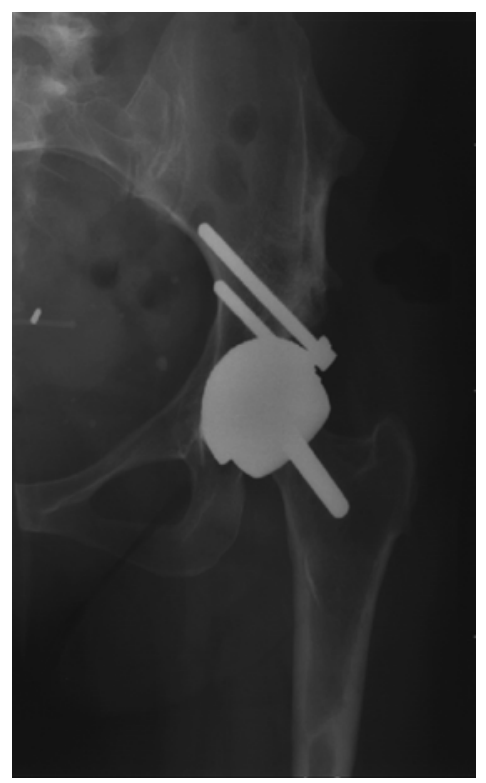

Fig. 1c

Anteroposterior radiographs of the left hip of a 42-year-old woman from the dysplasia group treated with a Birmingham Hip Resurfacing dysplasia cup and a contemporaneous femoral osteotomy a) pre-operative (Hartofilakidis grade II), b) three months post-operatively, and c) three years postoperatively (the dynamic compression plate was removed at one year after operation).

diagnosis recorded on the database by the operating surgeon where the causal mechanism for the development of OA was not known. Matching was performed with the ' $\mathrm{R}$ ' statistical package for age, gender, operating surgeon and date of operation between the developmental dysplasia group (cases) and the OA group (controls). ${ }^{13}$ This identified 96 hips (93 patients) matched with a diagnosis of primary OA. The mean age of the patients at the time of surgery was 43 years (14 to 65 ) in the dysplasia group and 47 years $(22$ to 76$)$ in the OA group. There were 18 procedures $(19 \%)$ in each group in men and $78(81 \%)$ in women. If the patient had a previous pelvic or femoral osteotomy, this was recorded. The indications for hip resurfacing were the same for both groups. A resurfacing was offered if there was painful end-stage joint degeneration in a patient of relatively young age and/or a high level of activity. Patients with renal impairment or a history of metal sensitivity were not treated by resurfacing. Severe deformation of the femoral head and neck was not a contraindication for resurfacing if it was anticipated that there would be adequate bone stock to support the femoral component following preparation of the femoral head. Cysts in the head or neck were not a reason for exclusion from resurfacing unless located at the junction of the femoral component and the superior femoral neck. All patients were counselled on the paucity of information about the risk of long-term exposure to raised levels of metal ions.

Procedures. The operations were performed by eight different surgeons. There were 45 procedures $(47 \%)$ in the dysplasia group performed by one of the authors $(\mathrm{JNOH})$, who also carried out $37(39 \%)$ of the operations in the OA group. Patients in both groups were placed in a lateral position and received an antiseptic pre-preparation in the anaesthetic room. The operations were performed in a laminar-airflow theatre. A posterolateral approach was used in all but three cases in each group. The direct lateral approach was used for these six cases, as it was the normal practice for one of the eight surgeons included in the study.

The size of the femoral component was determined by using a proprietary femoral neck measurer (Smith \& Nephew) with the greatest diameter found superoinferiorly. This in turn determined the size of the acetabular component to be used. If primary stable fixation of a standard acetabular component was unlikely to be achieved, a dysplasia component was used. In this situation some specific modifications to the procedure were made. The capsule was divided in the coronal plane at the superior acetabular margin, producing two flaps which were retracted anteriorly and posteriorly with stay sutures. The inferior margin of the true acetabulum was defined. The medial wall of the acetabulum can be relatively thick in acetabular dysplasia. Because of this, a test hole was often made in the floor of the acetabulum to assess the amount of bone available. Initially a small reamer was used to deepen the acetabulum followed by medialisation and occasional posterior reaming. Following the preparation of the acetabulum morcellised autograft obtained from the combined preparation of the femur and acetabulum was placed in the acetabulum and the acetabular component was impacted. The superior defect was filled with the morcellised graft, and the capsu- 
lar flaps were then sutured together above the socket to contain the graft.

Where a femoral osteotomy was necessary, the resurfacing was always done first. A shortening osteotomy was used to correct leg length and allow relocation where the hip presented in high subluxation or dislocation. The amount of bone to be resected was judged by attempting relocation of the resurfacing components and measuring the remaining gap. Where a normal thigh-foot axis angle was impossible to achieve, or the presence of femoral external rotation caused impingement of the femur on the ischium, the anteversion of the femur was also corrected to $20^{\circ}$ to $30^{\circ}$. The osteotomy was performed by laying a sixor seven-hole broad dynamic compression plate onto the femur immediately below the trochanteric flare on the lateral aspect. A transverse osteotomy was performed below the third or fourth hole and was usually $1 \mathrm{~cm}$ to $2 \mathrm{~cm}$ below the lesser trochanter. An example of a patient requiring contemporaneous femoral osteotomy is shown in Figure 1.

Details of the components used were kept, including their size and whether the dysplasia cup (Birmingham Hip Resurfacing System, Smith \& Nephew PLC) was used. Antibiotic prophylaxis was routinely $1.5 \mathrm{~g}$ cefuroxime on induction and two subsequent doses of $750 \mathrm{mg}$ cefuroxime. The thromboprophylaxis used was post-operative warfarin, in doses aiming to keep the international normalised ratio between 1.5 and 2.0. Post-operative rehabilitation was the same for both groups. Patients were allowed to bear full weight as soon as comfort dictated, and were advised to avoid contact sports for six months. Following this no restrictions were placed on activity.

Assessment of outcome. The Oxford hip score was used to assess pain and function. ${ }^{14}$ The scores were recorded preoperatively and at each subsequent outpatient visit. The patients were reviewed three times within the first year and thereafter on an annual or biannual basis up to five years, with the next planned review every five years. Where necessary, data were supplemented by a postal questionnaire and a review of the medical records. The cause of any death during follow-up was recorded. Failure was defined as the point where either the femoral or the acetabular component was revised. Where this occurred, details of the mode of failure and the findings at revision were recorded. Post-operative complications such as dislocations, infections, fractures, neurovascular injuries and re-operations were also noted. Symptomatic leg-length discrepancy was recorded.

Radiological assessment. Anteroposterior radiographs were obtained pre-operatively and at each subsequent postoperative review. The pre-operative degree of dysplasia was classified according to the classifications of Hartofilakidis, Stamos and Ioannidis ${ }^{15}$ and Crowe, Mani and Ranawat. ${ }^{16}$ The neck-to-shaft angle was measured and the presence of a cyst in the neck or head $>1 \mathrm{~cm}$ in diameter was noted. From the initial post-operative anteroposterior radiograph the acetabular abduction angle and the stem-shaft angle were recorded. The most recent follow-up radiograph was examined for signs of loosening. Radiolucent lines of $>2 \mathrm{~mm}$ around the acetabular component were recorded as described by DeLee and Charnley. ${ }^{17}$ On the femoral side the radiological evaluation of Amstutz et $\mathrm{al}^{8}$ was used. Evidence of thinning of the neck by $>10 \%$ at the latest followup was recorded, as was heterotopic bone formation according to the classification of Brooker et al. ${ }^{18}$

Statistical analysis. The ' $R$ ' statistical package was used, with confidence intervals derived from the two-sample $t$ test for continuous variables. The chi-squared test for dichotomous variables and the Mann-Whitney test were used where appropriate. ${ }^{13}$ Functional scores were assessed using the median and interquartile ranges. For the Oxford hip score, only those questionnaires with more than ten (of a possible 12) questions answered were considered valid, and the percentage of questions answered was taken as the final value. ${ }^{19}$ The Kaplan-Meier method for survival analysis and the Peto method ${ }^{13}$ for calculating the lower confidence limit for survival were used. A value of $\mathrm{p}<0.05$ was considered significant.

\section{Results}

Clinical. There was no significant difference between the two groups for age, gender, operating surgeon and postoperative follow-up. Details of the two groups are summarised in Table I. Complete follow-up data were available on 188 procedures $(97.9 \%)$. Three patients (four operations) were lost to follow-up but are included in all analyses up to the point of last follow-up. The follow-up for the dysplasia group was a mean of 4.4 years (2.0 to 8.5) and for the OA group a mean of 4.5 years $(2.2$ to 9.4$)$. Of the dysplasia cases $17(18 \%)$ were classified as Crowe grade III or IV. Of the dysplasia cases ten $(10 \%)$ had a previous acetabular osteotomy and six $(6 \%)$ a previous femoral osteotomy. The dysplasia cup was used in 34 cases $(35 \%)$ in the dysplasia group. None were used in the OA group. Nine different sizes of acetabular component were used in both groups, ranging from $44 \mathrm{~mm}$ to $62 \mathrm{~mm}$. The most common size in both groups was $52 \mathrm{~mm}$ (median $52 \mathrm{~mm}$ ). The most common size of femoral head was $46 \mathrm{~mm}$ (median $46 \mathrm{~mm}$ ) in both groups. Three cases (3\%) from the dysplasia group had a contemporaneous femoral osteotomy. All the osteotomies united. There were two deaths during follow-up in each group. None was related to the primary operation and all occurred between two and six years after surgery.

Survival. There were five revisions $(5.2 \%)$ in the dysplasia group and none in the OA group. Four of the revisions were for failure of the acetabular component. The dysplasia cup had been used in three of these cases. There were no septic failures. The revisions are summarised in Table II. The case that failed because of a fractured neck of femur was converted to a modular metal-on-metal cemented THR with retention of the uncemented resurfacing acetabular component. Three years and nine months after the first revision, 
Table I. Demographic and surgical factors for both groups

\begin{tabular}{|c|c|c|}
\hline & Developmental dysplasia group $(n=96)$ & Osteoarthritis group $(n=96)$ \\
\hline Mean age (range) & $43(14$ to 65$)$ & $47(21$ to 76$)$ \\
\hline \multicolumn{3}{|l|}{ Gender } \\
\hline $\mathrm{M}: \mathrm{F}$ & $18: 78$ & $18: 78$ \\
\hline \multicolumn{3}{|l|}{ Crowe grade } \\
\hline 1 & 40 & - \\
\hline II & 22 & - \\
\hline III & 12 & - \\
\hline IV & 5 & - \\
\hline$N / A^{*}$ & 17 & - \\
\hline \multicolumn{3}{|l|}{ Hartofilakidis grade } \\
\hline I & 39 & - \\
\hline$\|$ & 33 & - \\
\hline III & 7 & - \\
\hline $\mathrm{N} / \mathrm{A}$ & 17 & - \\
\hline \multicolumn{3}{|c|}{ Previous acetabular surgery } \\
\hline Yes & 10 & 0 \\
\hline No & 73 & 96 \\
\hline $\mathrm{N} / \mathrm{A}$ & 13 & 0 \\
\hline \multicolumn{3}{|c|}{ Previous femoral surgery } \\
\hline Yes & 6 & 0 \\
\hline No & 77 & 96 \\
\hline $\mathrm{N} / \mathrm{A}$ & 13 & 0 \\
\hline \multicolumn{3}{|l|}{ Dysplasia cup used } \\
\hline Yes & 34 & 0 \\
\hline No & 49 & 96 \\
\hline $\mathrm{N} / \mathrm{A}$ & 13 & 0 \\
\hline \multicolumn{3}{|l|}{ Failure } \\
\hline Femoral side & 1 & 0 \\
\hline Acetabular side & 4 & 0 \\
\hline
\end{tabular}

acetabular loosening was apparent and the patient underwent an acetabular revision. The Kaplan-Meier survival curves for the two groups are shown in Figure 2. A log-rank test demonstrated a significant difference in survival between the two groups $(\mathrm{p}=0.02)$. The five-year survival for 26 cases in the dysplasia group was $96.7 \%$ (95\% confidence interval (CI) 90 to 100) and 100\% (95\% CI 100 to 100) for 36 cases in the osteoarthritis group. A log-rank test showed no association between survival and the use of the dysplasia cup.

Functional scores. There were 71 patients from the dysplasia group who provided 239 Oxford hip scores, of which 200 were suitable for inclusion; 90 patients from the OA group provided 271 scores, of which 248 were suitable for inclusion. The pre-operative median Oxford hip score was $62.5 \%$ (interquartile range (IQR) 50.0 to 70.8 ) for the dysplasia group and $69.6 \%$ (IQR 52.1 to 79.2 ) for the OA group. Maximum improvement was reached by 12 months and was maintained for the duration of follow-up. The median Oxford hip score was $4.2 \%$ (IQR 0 to 6.3 ) for the dysplasia group and $2.1 \%$ (IQR 0.0 to 16.7 ) for the OA group at 12 months. There was no significant difference in the median Oxford hip score between the two groups at any time during the study.

Radiological. Of the dysplasia cases $83(86 \%)$ had radiographs which were available for analysis. The mean preoperative femoral neck-to-shaft angle was $145^{\circ}\left(108^{\circ}\right.$ to $\left.180^{\circ}\right)$ and $11(11 \%)$ had a cyst in the femoral head $>1 \mathrm{~cm}$. From the initial post-operative radiograph the mean stemto-shaft angle of the femoral component was $142^{\circ}\left(126^{\circ}\right.$ to $160^{\circ}$ ) placed in a mean of $3^{\circ}$ of varus in relation to the neck ( $28^{\circ}$ varus to $40^{\circ}$ valgus). The mean acetabular abduction angle was $47^{\circ}\left(25^{\circ}\right.$ to $\left.66^{\circ}\right)$. Of the 78 surviving cases with radiological evaluation (five were revised, one demonstrated loosening in all three DeLee and Charnley zones of the acetabular component at the most recent review. No cases demonstrated loosening of the femoral component. Of the 78 cases there were six $(8 \%)$ which demonstrated thinning of the neck $>10 \%$ in the dysplasia group. 
Table II. Details of each revision for the dysplasia group

\begin{tabular}{|c|c|c|c|c|c|c|c|}
\hline Revision & $\begin{array}{l}\text { Hartofilakidis } \\
\text { grade }\end{array}$ & Crowe grade & Gender & $\begin{array}{l}\text { Age at primary } \\
\text { operation (yrs) }\end{array}$ & $\begin{array}{l}\text { Acetabular } \\
\text { component (mm) }\end{array}$ & $\begin{array}{l}\text { Time to } \\
\text { revision (yrs) }\end{array}$ & Reason for revision \\
\hline 1 & II & II & $\mathrm{F}$ & 45 & 46 standard cup & 0.1 & Fractured neck of femur \\
\hline 2 & 1 & 1 & $\mathrm{~F}$ & 54 & 52 standard cup & 0.8 & $\begin{array}{l}\text { Aseptic loosening acetabular } \\
\text { component }\end{array}$ \\
\hline 3 & 1 & 1 & $\mathrm{~F}$ & 58 & 54 dysplasia cup & 2.7 & $\begin{array}{l}\text { Nonunion of ischium post triple } \\
\text { pelvic osteotomy: cup loose }\end{array}$ \\
\hline 4 & 1 & I & $\mathrm{F}$ & 42 & 50 dysplasia cup & 5.8 & $\begin{array}{l}\text { Post-traumatic fracture of } \\
\text { dysplasia acetabular } \\
\text { component screw: cup loose }\end{array}$ \\
\hline 5 & 1 & I & $\mathrm{F}$ & 54 & 50 dysplasia cup & 6.1 & $\begin{array}{l}\text { Aseptic loosening of acetabular } \\
\text { component }\end{array}$ \\
\hline
\end{tabular}

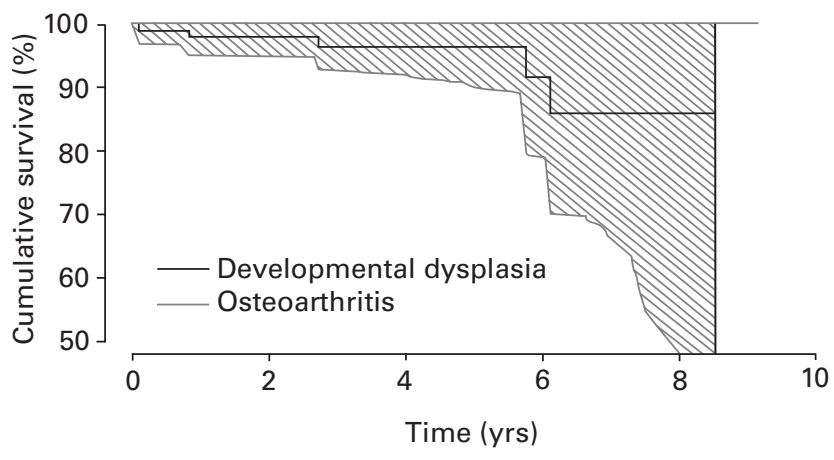

Fig. 2

Kaplan-Meier survival curves for the group with developmental dysplasia and the matched group with primary osteoarthritis following Birmingham Hip Resurfacing (survival to revision for any reason). The dashed area represents the $95 \%$ confidence intervals.

Complications. A suspected deep infection occurred at three months in one patient from the dysplasia group who had staged bilateral hip resurfacings. An open irrigation and debridement was performed. The intra-operative samples sent for bacteriology showed no growth and the patient recovered well without any further complaint. There was one dislocation in the dysplasia group in a patient in whom the component rotated into a vertical position following aseptic acetabular loosening; revision to a THR was carried out. Three patients from the dysplasia group were left with a symptomatic leg-length discrepancy of more than $1 \mathrm{~cm}$ post-operatively. All were longer on the operated side and required a shoe raise. There were no symptomatic deepvein thromboses or pulmonary embolic events.

\section{Discussion}

The long-term (10- to 20-year) survival rates of THR in developmental dysplasia vary widely, with reported rates between $69 \%$ and $100 \%{ }^{6,20-24}$ It has been suggested that a higher revision rate is related to the degree of dysplasia ${ }^{25}$ and placement of the acetabular component superiorly to produce a high hip centre. ${ }^{26}$ One of the advantages of hip resurfacing over conventional THR is the preservation of femoral bone stock. Hip resurfacing also avoids the difficulty sometimes encountered in patients with dysplasia during THR of a narrow and often curved proximal femoral canal.

The indications for the use of hip resurfacing in this study were broad, as is seen by the number of high-grade dysplasias, the wide range of pre-operative femoral neckshaft angles $\left(108^{\circ}\right.$ to $\left.180^{\circ}\right)$, and with $11 \%$ of cases having femoral cysts larger than $1 \mathrm{~cm}$.

The developers of modern hip resurfacing arthoplasty recommend placing the femoral component in slight valgus. Biomechanical studies also suggest that a relative valgus placement is beneficial to avoid fracture, in particular a minimum of $10^{\circ}$ of valgus for varus native necks. ${ }^{27}$ Placement of the femoral component in an ideal position can be technically demanding in patients with hip dysplasia, and in our series there was a wide range of varus to valgus positioning of the component relative to the centre of the femoral neck. We also included three patients who required a contemporaneous femoral osteotomy, which did not add any additional morbidity or mortality.

Femoral failure after hip resurfacing was rare $(1 \%)$ in our series and comparable to other reports where the Birmingham Hip Resurfacing has been used for dysplasia. ${ }^{12}$ In contrast, there have been reports of other resurfacing systems used in dysplasia where there have been five femoral failures in 59 hips $(8.4 \%){ }^{3}$ If femoral failure does occur, the preservation of femoral bone stock at the primary procedure allows the surgeon to proceed to a straightforward conversion to a THR. The functional outcome of revision of hip resurfacing to THR secondary to femoral failure has been demonstrated to be comparable to that of a primary THR. ${ }^{28}$ In our study the one failure due to an early fracture of the neck of the femur was converted to a cemented femoral component and the well-fixed acetabular component was left in situ. Following the revision the patient had resolution of pain, and returned to full activities. Unfortunately, 45 months later, she presented with acetabular loosening requiring further revision. It was only subsequent to the patient's primary surgery at the age of 45 that it 
became apparent that when she was 36 she had a surgically-induced early menopause for treatment of a gynaecological malignancy. At the time of surgery, she was on hormone replacement therapy, but suffered with symptoms indicative of under-treatment, and in retrospect it may be that the dose was inadequate. Perhaps early menopause should be considered a relative contraindication to hip resurfacing.

There is evidence to suggest that restoration of the hip centre is important to improve implant survival. ${ }^{26}$ Restoration of the normal hip centre was achieved in all patients in this series. However, there were four revisions secondary to failure of the acetabular component, and a further case which has radiological signs of failure of the acetabular component.

All of the acetabular failures occurred in low grades of dysplasia. One had a previous interlocking triple pelvic osteotomy. Another had a previous Chiari osteotomy, an operation notorious for causing problems in acetabular fixation. Although all of these sockets had been bone grafted using morcellised autograft, the quantity of bone packed behind the acetabular component was possibly inadequate to the task. In contrast, there were no acetabular failures in the high grades of dysplasia and the use of the dysplasia cup with two-screw fixation appears to provide the necessary stability for good medium-term results in this subgroup of patients. None had undergone previous acetabular surgery, and the surgeon may have benefited from the ability to fashion the thick medial floor into a secure foundation for the acetabular component.

The median size of the components was the same $(46 \mathrm{~mm}$ femoral and $52 \mathrm{~mm}$ acetabular) in both groups. Removal of excess acetabular bone was not found to be a problem, as the size of the components matched the natural size for the patient. The same median sizes found in the two groups are likely to be a reflection of the matching process for gender.

Because of the complexity of the operation, it would be expected that patients with dysplasia would have worse results than the matched group with primary OA. This may be due to the inherently unstable acetabulum in hip dysplasia. The implant survival of $96.7 \%$ at five years for all levels of dysplasia in our series is similar to the survival rates for THR for patients with dysplasia. ${ }^{6,20-24}$ This dropped to $85.9 \%$ (95\% CI 66.8 to 100$)$ at seven years (Fig. 2). The two failures which occurred after the five-year mark were in patients operated upon early in the surgeon's experience. Longer follow-up of a large number of patients is required to clarify the actual long-term survival rate of hip resurfacing in patients with dysplasia.

The reduction in pain and improvement of function as measured by the Oxford hip score was similar in both groups, with the lower scores being achieved between six and 12 months. This is better than in THR, where rehabilitation in dysplasia patients may take up to 18 months. ${ }^{29}$ Also, lifelong restrictions on activity are then often placed on young patients with THR in an attempt to reduce the wear expected in metal-on-polyethylene bearings. ${ }^{29}$ No such restrictions were placed on any of our patients, allowing them to return to high levels of activity.

A further advantage of hip resurfacing is the improved stability afforded by the large-diameter bearing. In this study the dislocation rate for the developmental dysplasia group was $1 \%$, which is comparable with the $5 \%$ quoted for hip resurfacing in the literature. ${ }^{3,12}$ The dislocation rates in THR for patients with developmental dysplasia range between $0 \%$ and $11 \% .{ }^{23,30,31}$

There are limitations to this study. The relatively short period of follow-up does not allow us to draw firm conclusions about the success of hip resurfacing in this challenging and often young group of patients in the long term, and in the OA group there are likely to be a number of cases which might have been classified, earlier in the disease process, as mild dysplasia (Crowe or Hartofilakidis grade I). However, none of the dysplasia group could have been classified as 'typical', 'idiopathic' or 'primary' OA radiologically.

The medium-term results of Birmingham Hip Resurfacing in all grades of developmental dysplasia are encouraging, albeit worse than in a group of matched patients with OA treated in the same manner. The functional outcome is similar in the two groups and the prevalence of femoral failure and of dislocation is very low. Acetabular failures occurred in the dysplasia group but not in the OA group. Metal-on-metal hip resurfacing is a valuable option in treating developmental dysplasia.

\section{Supplementary Material}

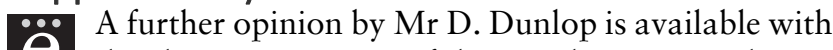
the electronic version of this article on our website at www.jbjs.org.uk

In support of the research for the preparation of this manuscript, one or more of the authors received grants from the British Hip Society (The McMinn Scholarship).

The author or one or more of the authors have received or will receive benefits for personal or professional use from a commercial party related directly or indirectly to the subject of this article. In addition, benefits have been or will be directed to a research fund, foundation, educational institution, or other nonprofit organisation with which one or more of the authors are associated.

\section{References}

1. Sugano N, Noble PC, Kamaric E, et al. The morphology of the femur in developmental dysplasia of the hip. J Bone Joint Surg [Br] 1998;80-B:711-19.

2. Noble PC, Kamaric E, Sugano N, et al. Three-dimensional shape of the dysplastic femur: implications for THR. Clin Orthop 2003;417:27-40.

3. Amstutz HC, Antoniades JT, Le Duff MJ, et al. Results of metal-on-metal hybrid hip resurfacing for Crowe type-I and II developmental dysplasia. J Bone Joint Surg [Am] 2007;89-A:339-46.

4. Charnley J, Feagin JA. Low-friction arthroplasty in congenital subluxation of the hip. Clin Orthop 1973;91:98-113.

5. Jasty M, Anderson MJ, Harris WH. Total hip replacement for developmental dysplasia of the hip. Clin Orthop 1995;311:40-5.

6. Perka C, Fischer U, Taylor WR, Matziolis G. Developmental hip dysplasia treated with total hip arthroplasty with a straight stem and a threaded cup. J Bone Joint Surg [Am] 2004;86-A:312-19.

7. Davlin LB, Amstutz HC, Tooke SM, Dorey FJ, Nasser S. Treatment of osteoarthrosis secondary to congenital dislocation of the hip: primary cemented surface replacement compared with conventional total hip replacement. J Bone Joint Surg [Am] 1990;72-A:1035-42. 
8. Amstutz HC, Beaulé PE, Dorey DJ, et al. Metal-on-metal hybrid surface arthroplasty: two to six-year follow-up study. J Bone Joint Surg [Am] 2004;86-A:28-39.

9. Treacy RB, McBryde CW, Pynsent PB. Birmingham hip resurfacing arthroplasty: a minimum follow-up of five years. J Bone Joint Surg [Br] 2005;87-B:167-70.

10. Daniel J, Pynsent PB, McMinn DJW. Metal-on-metal resurfacing of the hip in patients under the age of 55 years with osteoarthritis. J Bone Joint Surg [Br] 2004;86B:177-84.

11. Pollard TC, Baker RP, Eastaugh-Waring SJ, Bannister GC. Treatment of the young active patient with osteoarthritis of the hip: a five- to seven-year comparison of hybrid total hip arthroplasty and metal-on-metal resurfacing. J Bone Joint Surg [Br] 2006;88-B:592-600.

12. Nishii T, Sugano N, Miki H, et al. Five-year results of metal-on-metal resurfacing arthroplasty in Asian patients. J Arthroplasty 2007;22:176-83.

13. No authors listed. $R$ Development Core Team. A language and environment for statistical computing. R Foundation for Statistical Computing, Vienna, 2006 ISBN 3900051-07-0. http://www.R-project.org (date last accessed 28 January 2008).

14. Dawson J, Fitzpatrick R, Carr A, Murray D. Questionnaire on the perceptions of patients about total hip replacement. J Bone Joint Surg [Br] 1996;78-B:185-90.

15. Hartofilakidis G, Stamos K, loannidis TT. Low friction arthroplasty for old untreated congenital dislocation of the hip. J Bone Joint Surg [Br] 1988;70-B:182-6.

16. Crowe JF, Mani VJ, Ranawat CS. Total hip replacement in congenital dislocation and dysplasia of the hip. J Bone Joint Surg [Am] 1979;61-A:15-23.

17. DeLee JG, Charnley J. Radiological demarcation of cemented sockets in total hip replacement. Clin Orthop 1976;121:20-32.

18. Brooker AF, Bowerman JW, Robinson RA, Riley LH Jr. Ectopic ossification following total hip replacement: incidence and a method of classification. J Bone Joint Surg [Am] 1973;55-A:1629-32.

19. Pynsent PB, Adams DJ, Disney SP. The Oxford hip and knee outcome questionnaires for arthroplasty. J Bone Joint Surg [Br] 2005;87-B:241-8.

20. Hendrich C, Mehling I, Sauer U, Kirschner S, Martell JM. Cementless acetabular reconstruction and structural bone-grafting in dysplastic hips. J Bone Joint Surg [Am] 2006;88-A:387-94.
21. de Jong PT, Haverkamp D, van der Vis HM, Marti RK. Total hip replacement with a superolateral bone grafting for osteoarthritis secondary to dysplasia: a long-term follow-up. J Bone Joint Surg [Br] 2006;88-B:173-8.

22. Klapach AS, Callaghan JJ, Miller KA, et al. Total hip arthroplasty with cement and without acetabular bone graft for severe hip dysplasia: a concise follow-up, at a minimum of twenty years, of a previous report. J Bone Joint Surg [Am] 2005;87A:280-5.

23. lida H, Matsusue Y, Kawanabe K, et al. Cemented total hip arthroplasty with acetabular bone graft for developmental dysplasia: long-term results and survivorship analysis. J Bone Joint Surg [Br] 2000;82-B:176-84.

24. Bobak P, Wroblewski BM, Siney PD, Fleming PA, Hall R. Charnley low-friction arthroplasty with an autograft of the femoral head for developmental dysplasia of the hip: the 10- to 15-year results. J Bone Joint Surg [Br] 2000;82-B:508-11.

25. Kim Y-H, Kim J-S. Total hip arthroplasty in adult patients who had developmental dysplasia of the hip. J Arthroplasty 2005;20:1029-36.

26. Pagnano W, Hanssen AD, Lewallen DG, Shaughnessy WJ. The effect of superior placement of the acetabular component on the rate of loosening after total hip arthroplasty. J Bone Joint Surg [Am] 1996;78-A:1004-14.

27. Davis ET, OIsen M, Zdero R, Waddell JP, Schemitsch EH. Predictors of femoral neck fracture following hip resurfacing: a cadaveric study. Procs American Academy of orthopaedic surgeons. http://www.aaos.org/education/anmeet/anmt2007/ podium/podium.cfm?Pevent=127 (date last accessed 28 January 2008).

28. Ball ST, Le Duff MJ, Amstutz HC. Early results of conversion of failed femoral component in hip resurfacing arthroplasty. J Bone Joint Surg [Am] 2007;89-A:735-41.

29. Numair J, Joshi AB, Murphy JC, Porter ML, Hardinge K. Total hip arthroplasty for congenital dysplasia or dislocation of the hip: survivorship analysis and long-term results. J Bone Joint Surg [Am] 1997;79-A:1352-60.

30. MacKenzie JR, Kelley SS, Johnston RC. Total hip replacement for coxarthrosis secondary to congenital dysplasia and dislocation of the hip: long-term results. $J$ Bone Joint Surg [Am] 1996;78-A:55-61.

31. Inao S, Matsuno T. Cemented total hip arthroplasty with autogenous acetabular bone grafting for hips with developmental dysplasia in adults: the results at a minimum of ten years. J Bone Joint Surg [Br]2000;82-B:375-7. 